\title{
DESEMPENHO TÉRMICO E ACÚSTICO DE PAINÉIS DE FECHAMENTO EM MULTICAMADAS
}

\section{THERMAL AND ACOUSTIC PERFORMANCE CLOSING MULTILAYER PANELS}

\author{
Rovadávia Aline de Jesus Ribas', Henor Artur de Souza², Josimar Junio Adriano ${ }^{3}$, \\ Daniel José Rocha Pereira ${ }^{4}$
}

Recebido em 23 de novembro de 2012; recebido para revisão em 10 de dezembro de 2012; aceito em 10 de janeiro de 2013; disponível on-line em 11 de fevereiro de 2013.

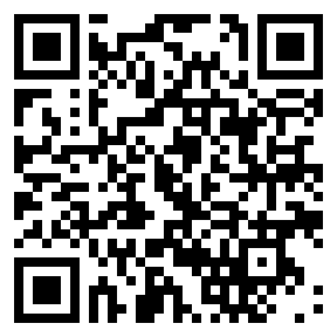

PALAVRAS CHAVES:

Painéis de fechamento;

Desempenho térmico;

Desempenho acústico;

Simulação numérica ESP-r.

\section{KEYWORDS:}

Closing panels;

Thermal performance; Acoustic performance; Numerical simulation ESP-r.

\section{* Contato com os autores:}

${ }^{1}$ e-mail : roviaaline@gmail.com (R. A. de J. Ribas)

Doutoranda do Programa de Pós-Graduação em Eng. Civil da Escola de Minas da Universidade Federal de Ouro Preto (UFOP/EM/PROPEC)

2e-mail : henor@em.ufop.br (H. A. de Souza)

Prof. do Dep. de Eng. de Controle e Automação e Técnicas Fundamentais e do Programa de Pós-Graduação em Engenharia Civil, Área:

Construção Metálica, da Escola de Minas da Universidade Federal de Ouro Preto (UFOP/EM/DECAT/PROPEC)

${ }^{3}$ e-mail : josimarjunioradriano@hotmail.com (J. J. Adriano)

Graduando do Curso de Engenharia Civil da Escola de Minas da Universidade Federal de Ouro Preto (UFOP/EM/DECIV)

${ }^{4}$ e-mail : danielrochapereira@yahoo.com.br (D. J. R. Pereira)

Graduando do Curso de Engenharia Civil da Escola de Minas da Universidade Federal de Ouro Preto (UFOP/EM/DECIV) 


\section{INTRODUÇÃO}

De modo geral, a construção estruturada em aço apresenta grande espaço para desenvolvimento, prometendo racionalização e atendimento às exigências de conservação de energia e conforto humano, apontando para projetos que apresentam desempenho global adequado. No entanto, a rapidez de montagem dos sistemas construtivos em estrutura metálica exige a aplicação de sistemas de fechamento que possuam a mesma filosofia de pré-fabricação e velocidade de construção. Os sistemas de fechamento industrializados apresentam-se como solução racional para edificações estruturadas em aço e a sua escolha deve ser feita de maneira criteriosa, considerando os diferentes tipos de fechamento disponibilizados. Os painéis utilizados possuem como vantagens a rapidez de montagem, a diminuição do peso da construção e ainda possibilitam soluções arquitetônicas criativas. Para que a sua escolha represente uma solução eficiente, é necessário que se leve em conta tanto os detalhes construtivos, quanto a capacidade de desempenho térmico e acústico desses fechamentos.

Esses sistemas de fechamento, por serem mais leves e de montagem rápida, têm capacidade de isolamento térmico e acústico questionável e necessitam de mais conhecimento quanto ao seu desempenho. 0 sistema de fechamento em multicamadas com a utilização de material absorvedor acústico, como a lã de vidro, na cavidade de ar entre as placas do fechamento, pode se mostrar eficiente no desempenho térmico e acústico, proporcionando conforto aos usuários (SALES, NEVES e SOUZA, 2001; GARCIA, 2004; RIBAS e SOUZA, 2011).

O objetivo desse trabalho é fazer uma avaliação do desempenho térmico e acústico de sistemas de fechamento aplicados em edificações estruturadas em aço, compostos por painéis aplicados em multicamadas, intermediados por uma camada de ar, contendo ou não material isolante na cavidade, tendo-se escolhido a lã de vidro (LVI). Esses sistemas de fechamento são constituídos por placa cimentícia (PLC), gesso cartonado (GEA), pré-moldado de concreto (PMC), concreto celular autoclavado (CCA) e poliestireno expandido (EPS).

O desempenho térmico e acústico de um ambiente depende da combinação de vários fatores, dentre os quais se destacam o posicionamento da edificação e suas dependências, a escolha e execução de paredes, pisos, tetos e esquadrias e a especificação de equipamentos e instalações. Todos os componentes são partes integrantes da edificação que funciona como um todo, não sendo possível determinar qual é mais relevante. Entretanto, pode se destacar a importância da especificação dos sistemas de fechamento verticais e horizontais, cuja constituição e montagem determinam, em grande parte, os níveis de desempenho térmico e acústico no ambiente construído.

\section{METODOLOGIA}

A avaliação do desempenho térmico é feita usando-se o software ESP-r (Energy Simulation Program - research), que, por meio de simulações computacionais, determina a temperatura e a umidade do ar no interior de uma edificação, utizando-se os dados climáticos da cidade de Belo Horizonte - MG como referência. A avaliação do desempenho acústico é realizada obtendo-se o tempo de reverberação em um recinto, aplicando-se uma formulação empírica, que usa em seus cálculos os valores de temperatura e umidade obtidos na avaliação térmica.

\subsection{AVALIAÇÃO DE DESEMPENHO TÉRMICO VIA SIMULAÇÃO TÉRMICA}

A simulação térmica é feita primeiramente, pois a temperatura e umidade do ar nos recintos, obtidas nessa etapa, contribuem para o cálculo da absorção sonora causada também pelo ar presente no ambiente considerado, que é utilizada na determinação do tempo de reverberação. Nas simulações, as condições térmicas da edificação são verificadas fazendo-se uma abordagem dinâmica que busca equacionar o equilíbrio entre perdas e ganhos de energia que ocorrem simultaneamente e variam ao logo de um dia.

As etapas para a análise do comportamento térmico de uma edificação via simulação numérica são:

- caracterização climática (temperatura do ar, umidade relativa do ar, radiação solar, direção e velocidade do vento);

- caracterização da edificação (posição geográfica, orientação, forma e volume, recinto típico: perfil de ocupação, equipamentos e iluminação);

- caracterização dos materiais de fechamento (dimensões, propriedades termofísicas);

- análise das interações térmicas e mássicas entre o meio externo e o meio interno;

- obtenção e avaliação da variação diária da temperatura e umidade do ar interno (edificação ventilada naturalmente).

\subsubsection{Caracterização das condições climáticas}

As condições climáticas são caracterizadas por valores horários de temperatura e da umidade relativa do ar e da radiação solar global e pela velocidade média 
do vento predominante nos dias típicos de verão e de inverno.

Para os dados climáticos, é aplicado o zoneamento climático apresentado pela norma NBR 15220 (ABNT, 2005). São adotados os dados de um dia típico de verão, da zona climática três, tomando-se a cidade de Belo Horizonte como referência (latitude: S $19^{\circ}$ 51'; longitude: W 43 57'; fuso horário: 3h; altitude: 785 $\mathrm{m})$. Os valores médios desse dia típico são apresentados na Tabela 1.

Um dia típico de verão, com frequência de ocorrência igual a 10\%, representa um dia tal que $10 \%$ dos dias do período de verão possuem temperatura máxima diária maior ou igual à temperatura desse dia. Da mesma forma, dia típico de inverno, com frequência de ocorrência igual a 10\%, representa um dia tal que $10 \%$ dos dias do período de inverno possuem temperatura mínima diária menor ou igual à temperatura desse dia (AKUTSU, 1998).

\subsubsection{Caracterização da edificação e dos fechamentos}

Propõe-se um modelo geométrico da edificação, baseado em zonas térmicas. Em seguida essa edificação é caracterizada por suas variáveis arquitetônicas, seus materiais construtivos e correspondentes camadas. A edificação é ventilada naturalmente e o desempenho térmico é avaliado em função do perfil obtido de temperatura e umidade internas.

A edificação projetada para as simulações possui área de $54 \mathrm{~m}^{2}$, está dividida em quatro zonas térmicas denominadas de sala, dormitório, banheiro e ático, pé direito de 3,0 $\mathrm{m}$ e: altura total de $4,20 \mathrm{~m}$. Na Figura 1 mostram-se a planta baixa e uma perspectiva da edificação geradas pelo ESP-rr, bem como sua orientação. Para configurar os ambientes, os fechamentos internos e externos são alterados durante as simulações, aplicandose os fechamentos conforme mostrados no Quadro 1.

Tabela 1: Valores médios do dia típico de verão considerado de Belo Horizonte.

\begin{tabular}{|c|c|c|c|c|c|}
\hline \multirow[b]{2}{*}{$\begin{array}{c}\text { Variação da } \\
\text { radiação solar } \\
\text { incidente em } \\
\text { plano horizontal } \\
\left(\mathrm{W} / \mathrm{m}^{2}\right)\end{array}$} & \multirow[b]{2}{*}{$\begin{array}{c}\text { Temperatura } \\
\text { de bulbo seco } \\
\left({ }^{\circ} \mathrm{C}\right)\end{array}$} & \multirow[b]{2}{*}{$\begin{array}{l}\text { Radiação solar total } \\
\text { incidente na direção } \\
\text { normal } \\
\left(\mathrm{W} / \mathrm{m}^{2}\right)\end{array}$} & \multicolumn{2}{|c|}{ Vento } & \multirow[b]{2}{*}{$\begin{array}{c}\text { Umidade } \\
\text { relativa do ar } \\
\text { (\%) }\end{array}$} \\
\hline & & & $\begin{array}{c}\text { Velocidade } \\
\qquad(\mathrm{m} / \mathrm{s})\end{array}$ & $\begin{array}{c}\text { Direção } \\
\text { (graus no } \\
\text { sentido horário } \\
\text { do Norte) }\end{array}$ & \\
\hline 97,7 & 24,5 & 299,1 & 1,7 & 77,5 & 63,1 \\
\hline
\end{tabular}
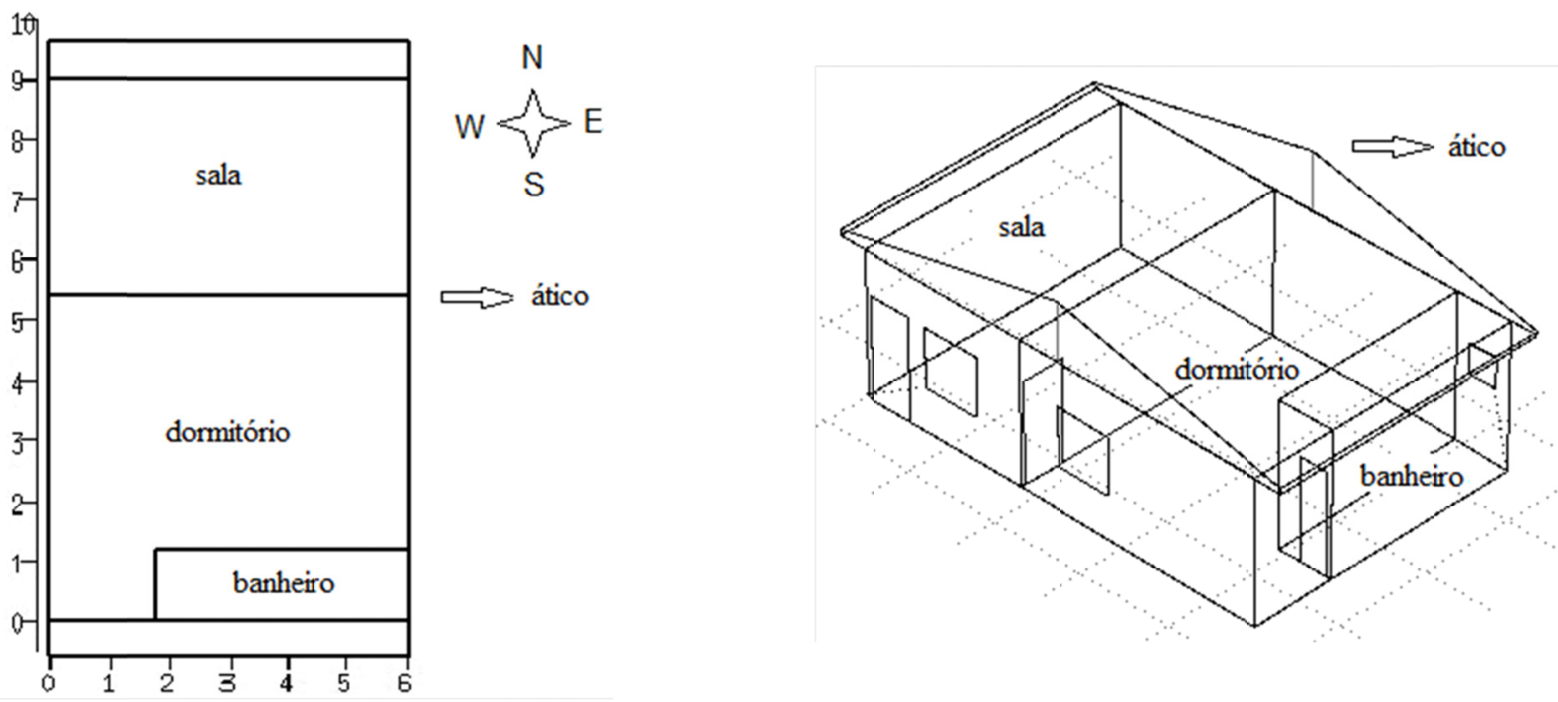

Figura 1: Planta baixa e perspectiva da edificação geradas pelo ESP-r. 
O uso do gesso acartonado como fechamento externo é somente conceitual. Mesmo assim, adota-se esse sistema de fechamento nas simulações para fins de comparação entre as várias situações. Definindo-se uma edificação padrão são fixados os seguintes parâmetros:

- piso em material cerâmico, sobre contrapiso de argamassa e base de concreto;

- cobertura em laje de concreto celular autoclavado, revestida internamente com argamassa, e telha de cerâmica, sendo que a cumeeira está a 1,0 m acima da laje;

- janelas em vidro comum transparente de 4 $\mathrm{mm}$ de espessura;

- portas externas e internas de $25 \mathrm{~mm}$ de espessura, em madeira de massa específica média e em compensado, respectivamente;

- fechamento do banheiro constituído de uma parede composta por placa cimentícia, ar, placa cimentícia e acabamento interno em cerâmica esmaltada.

As características físicas das zonas térmicas da edificação e as propriedades termofísicas dos elementos que compõem os sistemas de fechamento estão mostrados nas Tabelas 2 e 3, respectivamente. Não são consideradas no interior da edificação a presença de pessoas, lâmpadas acesas e equipamentos para o ganho de calor casual (sensível e latente). Para o fluxo de ar é adotada uma taxa de renovação igual a 3 ren/h.

Quadro 1: Painéis analisados e espessuras das camadas.

\begin{tabular}{|l|l|}
\hline Material do fechamento e espessura $(\mathbf{m m})$ & Material do fechamento e espessura $(\mathbf{m m})$ \\
\hline PLC(12)-ar(75)-PLC(12) & GEA(15)-ar(75)-GEA(15) \\
\hline$P L C(12)-L V I(50)-\operatorname{ar}(25)-P L C(12)$ & GEA(15)-LVI(50)-ar(25)-GEA(15) \\
\hline$P L C(12)-\operatorname{ar}(75)-G E A(15)$ & PMC(75)-ar(75)-PMC(75) \\
\hline PLC(12)-LVI(50)-ar(25)-GEA(15) & PMC(75)-LVI(50)-ar(25)-PMC(75) \\
\hline EPS(100)-ar(75)-EPS(100) & CCA(100)-ar(75)-CCA(100) \\
\hline EPS(100)-LVI(50)-ar(25)-EPS(100) & CCA(100)-LVI(50)-ar(25)-CCA(100) \\
\hline
\end{tabular}

Tabela 2: Características físicas das zonas térmicas da edificação.

\begin{tabular}{ccccc} 
Item & Sala & Dormitório & Banheiro & Ático \\
\hline Área do piso $\left(\mathbf{m}^{\mathbf{2}}\right)$ & 21,6 & 27,4 & 5,04 & 61,2 \\
Área das paredes $\left(\mathbf{m}^{\mathbf{2}}\right)$ & 99,4 & 62,6 & 42,1 & 137 \\
Volume do recinto $\left(\mathbf{m}^{\mathbf{3}}\right)$ & 64,8 & 82,1 & 15,1 & 17,3 \\
Área constituída por portas $\left(\mathbf{m}^{2}\right)$ & 3,78 & 3,15 & 1,26 & 0,00 \\
Área constituída por vidros/janelas $\left(\mathbf{m}^{2}\right)$ & 1,44 & 1,44 & 0,36 & 0,00 \\
\end{tabular}


Tabela 3: Propriedades termofísicas dos elementos componentes dos sistemas de fechamento.

\begin{tabular}{cccccc} 
Material & $\rho\left(\mathrm{kg} / \mathrm{m}^{3}\right)$ & $k(\mathrm{~W} /(\mathrm{m} \mathrm{K}))$ & $c_{p}(\mathrm{~J} /(\mathrm{kg} \mathrm{K}))$ & $\varepsilon$ & $\alpha$ \\
\hline EPS (revestido por argamassa) & 2100 & 1,15 & 1000 & 0,900 & 0,500 \\
Concreto celular autoclavado & 500 & 0,17 & 1000 & 0,900 & 0,650 \\
Gesso acartonado & 750 & 0,35 & 840 & 0,900 & 0,500 \\
Lã de vidro & 100 & 0,045 & 700 & 0,900 & 0,500 \\
Placa cimentícia & 1330 & 0,35 & 1050 & 0,960 & 0,600 \\
Pré-moldado de concreto & 2400 & 1,93 & 1000 & 0,900 & 0,700 \\
\hline
\end{tabular}

\subsection{TEMPO DE REVERBERAÇÃO}

O tempo de reverberação (TR) é definido pelo tempo necessário para que o nível de pressão sonora em um recinto decaia em $60 \mathrm{~dB}$, a partir da interrupção da fonte, e constitui um indicador que pode ser aplicado para determinar o desempenho acústico de um ambiente fechado. Essa taxa de atenuação do som, bem como a fração de energia sonora absorvida, depende da frequência do som emitido, da capacidade do material do contorno para absorver essa frequência, do volume de ar e do espectro de frequências do som. O tempo de reverberação inclui a absorção devida ao fechamento, ao mobiliário, aos ocupantes e ao ar presente (BIES e HANSEN, 2003; HASSAN, 2009; MAEKAWA, RINDEL e LORD, 2011).

Quanto maior a absorção total do recinto, menor é a energia sonora do campo reverberante. Diante disso, para o controle do ruído proveniente da reverberação no local, é necessário conhecer a capacidade dos sistemas de fechamento e dos materiais aplicados no interior da edificação em absorver o ruído interno. A absorção sonora representa a capacidade de um material de absorver a energia sonora, ocorrendo em graus variados, sendo determinada pelo coeficiente de absorção sonora dos materiais envolvidos $\left(\alpha_{i}\right)$. 0 conhecimento do coeficiente de absorção sonora é necessário para a determinação da área de absorção equivalente do fechamento, ocupantes, objetos e ar no interior de um recinto, que é item preponderante no cálculo do tempo de reverberação. Esse coeficiente consiste na fração da energia sonora incidente que é absorvida pelo material e depende da frequência do som e do ângulo de incidência das ondas sonoras no material (CITHERLET, 2001; HASSAN, 2009).

Segundo Wallace Clement Sabine o tempo de reverberação pode ser dado por (CITHERLET, 2001; BISTAFA, 2006):

$$
T R=0,161 \cdot \frac{V}{A_{f}^{t}}
$$

Onde:

TR: tempo de reverberação (s);

$\mathrm{V}$ : volume do recinto $\left(\mathrm{m}^{3}\right)$;

$f$ : frequência considerada $(\mathrm{Hz})$;

$\mathrm{A}_{\mathrm{f}}^{\mathrm{t}}$ : área total equivalente do recinto para a frequência $f\left(\mathrm{~m}^{2}\right.$ Sabine).

A área total equivalente do recinto $\left(A_{f}^{t}\right)$ inclui a absorção sonora devida ao sistema de fechamento, ao mobiliário, aos ocupantes presentes, e ao ar ambiente, dada por:

$$
A_{f}^{t}=A_{f}^{f e c h}+A_{f}^{o b j+p e s}+A_{f}^{a r}
$$

\section{Onde:}

$A_{f}^{t}$ : área total equivalente do recinto para a frequência $f$ ( $\mathrm{m}^{2}$ Sabine);

$A_{f}^{f e c h}$ : área de absorção equivalente do fechamento no interior do recinto ( $\mathrm{m}^{2}$ Sabine);

$A_{f}^{o b j+p e s}$ : área de absorção equivalente dos objetos e pessoas no interior do recinto ( $\mathrm{m}^{2}$ Sabine);

$A_{f}^{a r}$ : absorção equivalente devida ao ar no interior do recinto ( $\mathrm{m}^{2}$ Sabine).

Dentre as expressões propostas para o cálculo da área de absorção equivalente do fechamento no interior de um ambiente fechado, encontra-se a equação de Sabine, dada pelo produto exposto na Equação 03 (CITHERLET, 2001; HASSAN, 2009):

$$
A_{f}^{f e c h}=\sum_{i}^{f e c h}\left(S_{i} \cdot \alpha_{i, f}^{S a b}\right)
$$




\section{Onde:}

$A_{f}^{\text {fech }}$ : área de absorção equivalente do fechamento no interior do recinto ( $\mathrm{m}^{2}$ Sabine);

$S_{i}$ : área da superfície interna $i$ do recinto $\left(\mathrm{m}^{2}\right)$;

$\alpha_{i, f}^{S a b}$ : coeficiente de absorção de Sabine da superfície $i$ na frequência $f$.

A absorção sonora devida ao ar presente no recinto $\left(A_{f}^{a r}\right)$ pode ser expressa pela equação (KNUDSEN e HARRIS ${ }^{1}, 1978$ apud CITHERLET, 2001):

$$
A_{f}^{a r}=4 m . V
$$

Onde:

$A_{f}^{a r}$ : absorção equivalente devida ao ar no interior do recinto $\left(\mathrm{m}^{2}\right.$ Sabine);

$m$ : coeficiente de absorção sonora do $\operatorname{ar}\left(\mathrm{m}^{-1}\right)$; $\mathrm{V}$ : volume do recinto $\left(\mathrm{m}^{3}\right)$.

Pode-se expressar o coeficiente $m$ para condições específicas do ar aplicando-se as formulações apresentadas nas Equações 05 a 08 (CITHERLET, 2001):

$$
\begin{aligned}
& m=3,68.10^{-11} f^{2}\left(\frac{P}{P_{0}}\right)^{-1}\left(\frac{T}{T_{0}}\right)^{1 / 2}+\left(\frac{T}{T_{0}}\right)^{-5 / 2}\left(\begin{array}{l}
0,1068 \cdot e^{-3352,0 / T} \cdot \frac{2 f^{2}}{f_{r N}+\frac{f^{2}}{f_{r N}}}+ \\
+0,0128 \cdot e^{-2239,1 / T} \cdot \frac{2 f^{2}}{f_{r 0}+\frac{f^{2}}{f_{r 0}}}
\end{array}\right) \\
& f_{r N}=\frac{P}{P_{0}}\left(\frac{T}{T_{0}}\right)^{-1 / 2}\left(9+280 h_{a} \cdot e^{-4,170\left[\left(\frac{T}{T_{0}}\right)^{-1 / 3}-1\right]}\right) \\
& f_{r 0}=\frac{P}{P_{0}}\left(24+4,04 \cdot 10^{4} h_{a} \frac{0,02+h_{a}}{0,391+h_{a}}\right) \\
& h_{a}=\frac{h_{r} \cdot 10^{-6,8346\left(T_{0 \mathrm{i}} / T\right)^{1,261}+4,6151}}{\frac{P}{P_{0}}}
\end{aligned}
$$

Onde:

$m=$ : coeficiente de absorção sonora do $\operatorname{ar}\left(\mathrm{m}^{-1}\right)$;

$f$ : frequência considerada $(\mathrm{Hz})$;

$\mathrm{P}$ : pressão do ar $(\mathrm{kPa})$;

$\mathrm{P}_{0}$ : pressão de referência do ar $(101,325 \mathrm{kPa})$;

$\mathrm{T}$ : temperatura do ar (K);

$\mathrm{T}_{0}$ : temperatura de referência do ar $(298,15 \mathrm{~K})$;

$f_{r N}$ : frequência de relaxação para o Nitrogênio $(\mathrm{Hz})$;

$f_{r 0}$ : frequência de relaxação para o Oxigênio $(\mathrm{Hz})$;

$h_{a}$ : concentração molar do vapor de água (\%);

$h_{r}$ : umidade relativa do ar (\%);

$T_{0 i}=$ temperatura isotérmica de ponto triplo $(273,16 \mathrm{~K})$.

1 KNUDSEN V.O.; HARRIS C.M. Acoustical designing in architecture. The Acoustical Society of America ed. New York, 1978. 


\section{DESEMPENHO TÉRMICO E ACÚSTICO DE UMA EDIFICAÇÃO}

\subsection{AVALIAÇÃO DO DESEMPENHO TÉRMICO}

O desempenho térmico de uma edificação está relacionado a uma resposta adequada às condições climáticas e ao seu entorno e sua avaliação visa proporcionar condições de conforto térmico atendendo às exigências dos usuários e racionalizando o consumo de energía, por meio da análise da resposta global da edificação às trocas de calor e massa entre o ambiente interno e externo. No Brasil, para atender às exigências de desempenho térmico, a edificação deve possuir características que levem em consideração sua região de implantação, as respectivas características bioclimáticas e o comportamento interativo entre fachada, cobertura e piso, conforme propõe a norma NBR 15220 (ABNT, 2005).

A norma NBR 15575 (ABNT, 2008) apresenta condições térmicas no interior da edificação que sejam melhores ou iguais às do ambiente externo, à sombra, para o dia típico de verão, e que propiciem conforto térmico no interior da edlificação, no dia típico de inverno. Essa norma apresenta procedimentos de verificação do atendimento aos requisitos e critérios para fachadas e coberturas e para os sistemas de fechamento. O nível mínimo de desempenho, via simulação computacional ou medição in loco, deve atender os critérios conforme apresentados na Tabela 4.

Tabela 4: Painéis analisados e espessuras das camadas

\begin{tabular}{c|c|c|c}
\multirow{2}{*}{ Nível de desempenho } & Critério (verão) & \multicolumn{2}{|c}{ Critério (inverno) } \\
\cline { 2 - 4 } & Zonas 1 a 8 & Zonas 1 a 5 & Zonas 6 a 8 \\
\hline Mínimo & $T_{i, \text { máx }} \leq T_{e, \text { máx }}$ & $T_{i, \text { min }} \geq\left(T_{e, \text { min }}+3^{\circ} \mathrm{C}\right)$ & Não precisa ser verificado \\
\hline
\end{tabular}

\subsection{AVALIAÇÃO DO DESEMPENHO ACÚSTICO}

O tempo de reverberação deve estar de acordo com o uso do recinto, pois valores inadequados podem atrapalhar a inteligibilidade ou o entendimento da fala. Quanto maior o volume do recinto e a presença de materiais pouco absorvedores, maior é a duração da reverberação. Se a reverberação persistir no ambiente por muito tempo, pode haver sobreposição de sílabas e/ou notas musicais e se ela desaparecer imediata e totalmente, alguns tipos de fontes sonoras podem não ser percebidos (MAEKAWA, RINDEL e LORD, 2011).

Recintos destinados à palavra falada (salas de aula, de conferência e teatros) requerem tempos de reverberação mais curtos, para que o som refletido decaia rapidamente sem interferir no som direto nem reduzir sua inteligibilidade. No caso de edificações residenciais, onde é importante a comunicação, a reverberação deve ser equilibrada para o som ser assimilado com clareza e a análise é feita geralmente em ambientes que requerem maior conforto acústico, tais como quartos e salas. Para uma pequena sala, como é o caso aqui avaliado, é recomendado o valor de 0,5 s. Em salas de concerto esse tempo deve ser mais longo, pois a reverberação é em parte necessária para que haja qualidade acústica à música orquestral (MEHTA,
JOHNSON e ROCAFORT, 1999; BISTAFA, 2006).

\section{RESULTADOS E ANÁLISE}

Para a avaliação térmica, obtém-se a variação temporal da temperatura e umidade relativa do ar nas quatro zonas térmicas considerando-se os sistemas de fechamento propostos (Figura 2). Para a avaliação acústica, é calculado o tempo de reverberação das superfícies dos fechamentos (TR), sendo necessários os coeficientes de absorção sonora $\left(\alpha_{i}\right)$ dos materiais (BISTAFA, 2006; MAEKAWA; RINDEL; LORD, 2011; RILEM, 2005) aplicados no interior do recinto estudado (Figura 3). Os resultados obtidos para a sala na frequência de $1000 \mathrm{~Hz}$ são mostrados nas Figuras 4 a 9.

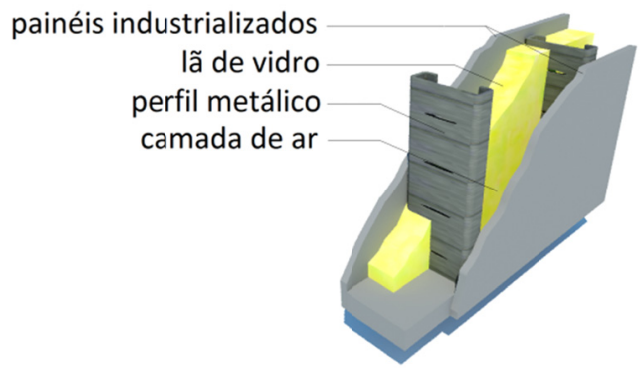

Figura 2: Esquema dos sistemas de fechamento aplicados na edificação. 


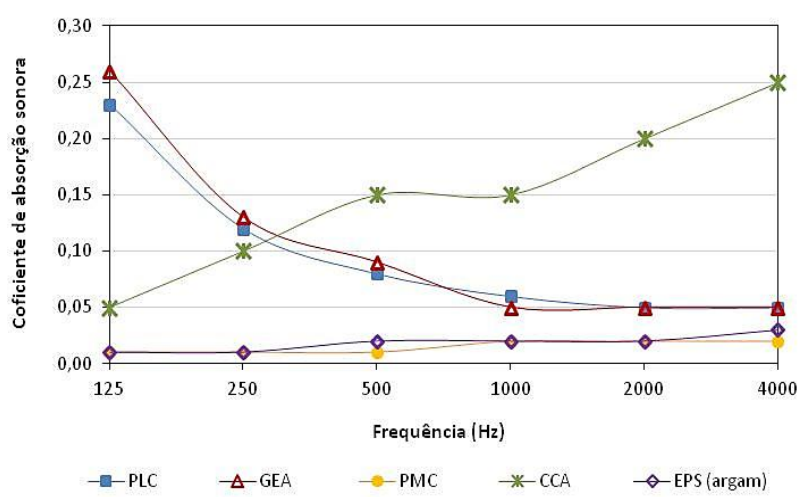

Figura 3: Coeficientes de absorção sonora dos materiais aplicados no interior dos recintos.

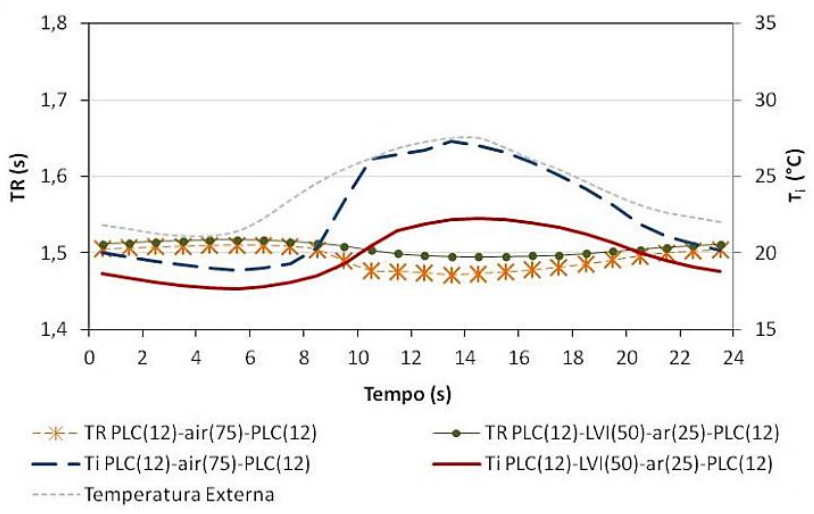

Figura 4: TR e $T_{i}$ em função do tempo - PLC.

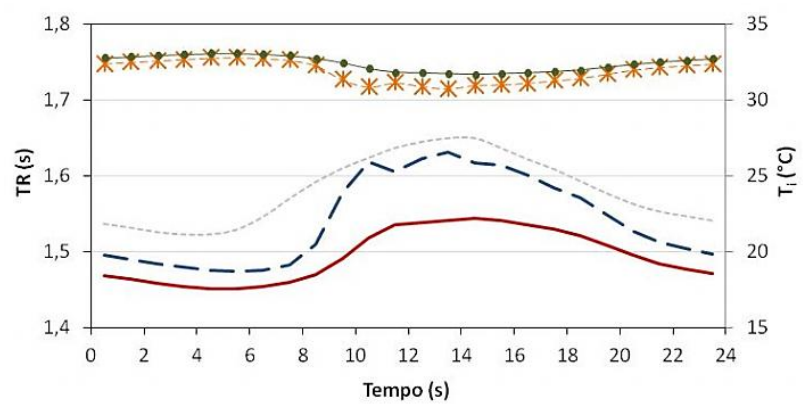

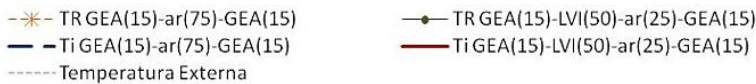

Figura 5: TR e $T_{i}$ em função do tempo - GEA.

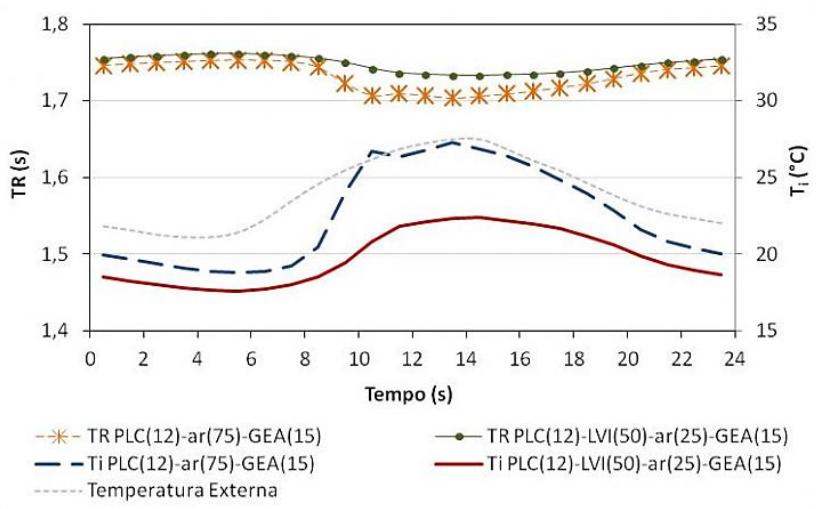

Figura 6: TR e Ti em função do tempo - PLC-GEA.

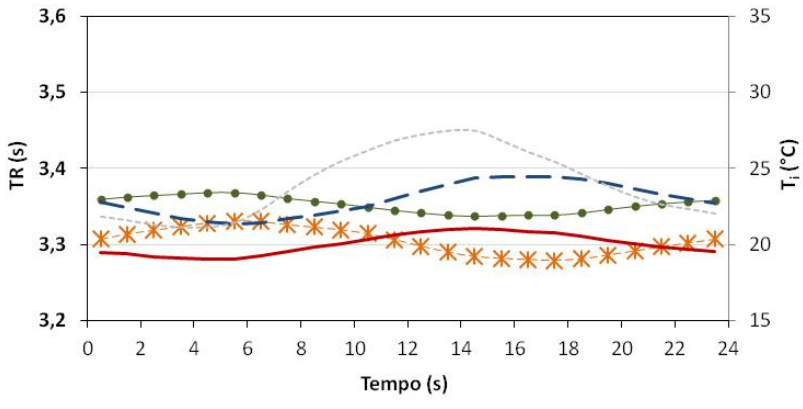

- * - TR PMC(75)-ar(75)-PMC(75) — TR PMC(75)-LVI(50)-ar(25)-PMC(75) - -Ti PMC(75)-ar(75)-PMC(75) - Ti PMC(75)-LVI(50)-ar(25)-PMC(75) Temperatura Externa

Figura 7: TR e Ti em função do tempo - PMC.
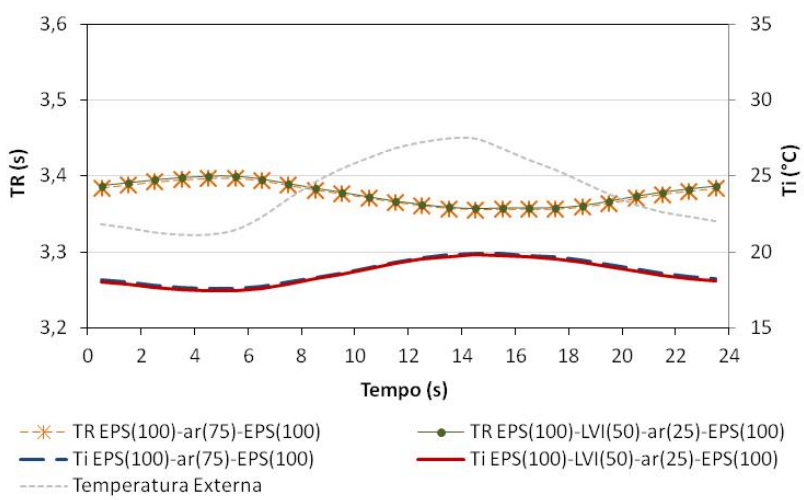

Figura 8: $T R$ e $T_{i}$ em função do tempo - EPS.

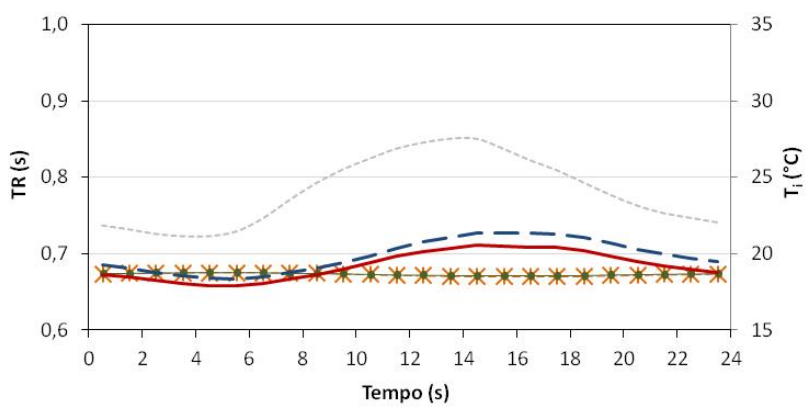
-* TRCCA(100)--ar(75)-CCA(100) $\quad \longrightarrow$ TRCCA(100)-LVI(50)--ar(25)-CCA(100)
- -TiCCA(100)-ar(75)-CCA(100) TiCCA(100)-LVI(50)-ar(25)-CCA(100)
- Temperatura Externa

Figura 9: TR e $T_{i}$ em função do tempo - CCA.

Em relação ao desempenho térmico, para as condições de verão, os fechamentos analisados atendem ao critério mínimo de desempenho térmico, ou seja, $\mathrm{T}_{\mathrm{i}, \text { máx }} \leq \mathrm{T}_{\mathrm{e} \text {,máx }}$. Para a maioria dos fechamentos recheados com lã de vidro resultam temperaturas internas mais baixas do que os mesmos fechamentos sem esse material, apresentando desempenho térmico melhor (Figuras 4 a 9). Considerando a temperatura externa máxima de $27,5{ }^{\circ} \mathrm{C}$, no horário de $13: 30 \mathrm{~h}$, dentre as composições de fechamento em GEA e PLC, para os fechamentos em PLC(12)-LVI(50)-ar(25)-PLC(12) e GEA(15)-LVI(50)-ar(25)-GEA(15) resultam a menor temperatura interna $\left(22,1^{\circ} \mathrm{C}\right)$ (Figuras 4 a 6$)$. Dentre as composições de fechamento em PMC, EPS e CCA, para o 
fechamento em EPS(100)-LVI(50)-ar(25)-EPS(100) temse a menor temperatura interna $\left(19,1{ }^{\circ} \mathrm{C}\right)$, no mesmo horário (Figuras 7 a 9). Assim, dentre todos os fechamentos analisados, esse último apresenta o melhor desempenho térmico (Figuras 4 a 9).

Em relação ao desempenho acústico, para a frequência considerada de $1000 \mathrm{~Hz}$, o CCA possui maior coeficiente de absorção sonora (Figura 3). Também para essa frequência, observa-se que os fechamentos não atendem o critério de tempo de reverberação no valor de 0,5 s. Mas, deve-se levar em conta que na edificação não está sendo considerada a presença de pessoas e mobiliário, o que tende a elevar o valor do tempo de reverberação (Figuras 4 a 9).

De modo geral, os fechamentos recheados com lã de vidro mostram um tempo de reverberação um pouco maior do que os outros fechamentos, em média $0,02 \mathrm{~s}$, nos horários de temperatura externa mais elevada (09:00-18:00). Esse fato se deve à redução do valor da umidade do ar nesse período e a consequente diminuição da absorção sonora devida ao ar presente no recinto. No caso dos fechamentos em EPS e CCA, a presença da lã de vidro na cavidade de ar praticamente não influenciou no tempo de reverberação. Dentre as composições de fechamento em GEA e PLC, o fechamento em PLC(12)-ar(75)-PLC(12) apresenta o menor tempo de reverberação $(1,48 \mathrm{~s})$, no horário de 13:30 (Figuras 4 a 6). Dentre as composições de fechamento em PMC, EPS e CCA, os fechamentos em CCA(100)-ar(75)-CCA(100) e CCA(100)-LVI(50)-ar(25)CCA(100) apresenta o menor tempo de reverberação (0,67 s), no mesmo horário (Figuras 7 a 9). Assim, dentre os fechamentos analisados, esse último apresenta o melhor desempenho acústico (Figuras 4 a 9).

\section{CONCLUSÕES}

Avalia-se o desempenho térmico e acústico dos painéis em multicamadas constituídos por placa cimentícia, gesso acartonado, pré-moldado de concreto, concreto celular autoclavado e poliestireno expandido. Os fechamentos analisados, quando recheados com lã de vidro, em geral, apresentam desempenho térmico satisfatório, mostrando que o sistema multicamadas com a aplicação de material isolante na cavidade de ar entre as paredes pode se apresentar como uma solução eficiente capaz de proporcionar conforto inclusão de pessoas e objetos no ambiente, obtendo-se uma melhor resposta acústica do ambiente.
Esse estudo mostra que é necessário verificar o desempenho térmico e acústico dos sistemas de fechamento propostos e a escolha de um ou de outro sistema dependerá do equilíbrio das respostas alcançadas. O método apresentado, que vincula avaliação térmica e acústica, por meio do software ESP-r, pode ser aplicado a qualquer tipo de edificação (residencial, comercial, industrial) devendo-se apenas modificar os dados climáticos e as características físicas, térmicas e acústicas dos materiais dos fechamentos da edificação.

\section{AGRADECIMENTOS}

Os autores agradecem o apoio financeiro da FAPEMIG e do CNPq a este trabalho.

\section{REFERÊNCIAS BIBLIOGRÁFICAS}

AKUTSU, M. Método para a avaliação do desempenho térmico de edificações no Brasil. 1998. Tese (Doutorado em Arquitetura). Universidade de São Paulo. São Paulo, 1998.

ASSOCIAÇÃO BRASILEIRA DE NORMAS TÉCNICAS (ABNT). NBR 15220: Desempenho térmico de edificações. Rio de Janeiro, 2005. $92 \mathrm{p}$.

ASSOCIAÇÃO BRASILEIRA DE NORMAS TÉCNICAS (ABNT). NBR 15575: Edifícios habitacionais de até 5 pavimentos: desempenho. Rio de Janeiro, 2008. 251 p.

BIES, D.A.; HANSEN, C. H. Engineering noise control: Theory and Practice. 3. ed. London e New York: Spon Press, 2003. $719 \mathrm{p}$.

BISTAFA, S.R. Acústica aplicada ao controle de ruído. São Paulo: Edgard Blücher, 2006. 368 p.

CITHERLET, S. Towards the holistic assessment of building performance based on an integrated simulation approach. 2001. Thesis (Architecture) - Lausanne: Swiss Federal Institute of Technology (EPFL), 2001. 164 p.

GARCIA, D. Análise do isolamento sonoro de sistemas construtivos residenciais estruturados em aço. Dissertação (Mestrado em Engenharia de Estruturas) - Pós-Graduação em Engenharia de Estruturas, Universidade Federal de Minas Gerais, Belo Horizonte, 2004. 117 p.

HASSAN, O.A.B. Building acoustics and vibrations: Theory and Practice. Singapore: World Scientific, 2009. 947 p.

MAEKAWA, Z; RINDEL, J.H.; LORD, P. Environmental and architectural acoustics. 2. ed. New York: Spon Press, 2011. $360 \mathrm{p}$. 
MEHTA, M.; JOHNSON, J.; ROCAFORT, J. Architectural acoustics: Principles and Design. New Jersey: Prentice Hall, 1999. 446 p.

RIBAS, R.A.J; SOUZA, H.A. Acoustic performance of closing panels used in steel structure buildings. In: INTERNATIONAL CONGRESS ON SOUND \& VIBRATION, 18., 2011, Rio de Janeiro, Proceedings... 2. ed. Rio de Janeiro: IIAV, 2011. p. 1-8.

RILEM - INTERNATIONAL UNION OF LABORATORIES AND EXPERTS IN CONSTRUCTION MATERIALS, SYSTEMS AND STRUCTURES. RILEM Technical Comittees 78-MCA and 51-ALC. Autoclaved aerated concrete: properties, testing and design. New York: Taylor \& Francis, 2005. ISBN 0419179607. Disponível em: <http://books.google.com.br/books?id=diiPFbTX9EC\&printsec $=$ frontcover $\& \mathrm{hl}=\mathrm{pt}-$

$B R \&$ source $=g b s$ ge_summary_r $\&$ cad $=0 \# v=$ onepage $\& q \& f=f a l s e>$. Acesso em: 5 abr. 2012. 456 p.

SALES, U.C.; NEVES, F.A.; SOUZA, H.A. Avaliação comparativa do desempenho acústico de painéis de vedação pré-fabricados. In: ENCONTRO NACIONAL SOBRE CONFORTO NO AMBIENTE CONSTRUÍDO, 4, e ENCONTRO LATINO AMERICANO SOBRE CONFORTO NO AMBIENTE CONSTRUÍDO, 3, 2001. v.1, p. 1-7. 